\title{
electronics
}

ISSN 2079-9292

www.mdpi.com/journal/electronics

New Book Received *

\section{Handbook of RF, Microwave, and Millimeter-Wave Components. Edited by Leonid A. Belov, Sergey M. Smolskiy and Victor N. Kochemasov, Artech House, 2012; 504 pages. Price: £119.00, ISBN 978-1-60807-209-5}

\author{
Shu-Kun Lin \\ MDPI AG, Kandererstrasse 25, CH-4057 Basel, Switzerland; E-Mail: lin@mdpi.com \\ Received: 9 January 2013 / Accepted: 10 January 2013 / Published: 14 January 2013
}

The following paragraphs are reproduced from the website of the publisher [1].

This unique and comprehensive resource offers you a detailed treatment of the operations principles, key parameters, and specific characteristics of active and passive RF, microwave, and millimeter-wave components. The book covers both linear and nonlinear components that are used in a wide range of application areas, from communications and information sciences, to avionics, space, and military engineering.

This practical book presents descriptions and clear examples of the best materials and products used in the field, including laminates, prepregs, substrates; microstrip, coaxial and waveguide transmission lines; fixed and rotating connectors; matching and adjusting elements; frequency filters; phase shifters; and ferrite gates and circulators. Moreover, the book offers you in-depth discussions on microwave switches and matrices, including MEMS technology, solid state and vacuum amplifiers, mixers, modulators and demodulators, and oscillation sources. You also find coverage of the stable frequency synthesizer structure and sources of modulated or noisy signals. Greatly adding to the usefulness of this volume is the inclusion of more than 700 Internet addresses of manufacturers from across the globe. 


\section{Table of Contents}

\section{Preface xi}

\section{Chapter 1 Devices for Signal Generation and Processing 1}

1.1 General Information About Signals 1

1.2 Architecture of Devices for Generation and Processing of Signals 6

1.2.1 Reference Oscillations 6

1.2.2 Signals with Phase Modulation and Shift Keying 10

1.2.3 QAM Signals 16

1.2.4 Signals with Frequency Modulation and Shift Keying 18

1.2.5 Multifrequency Signals 24

1.3 Requirements to Devices and Components 27

1.4 Product Certification and Quality Standards 30

1.5 Foundry Service 36

Questions 38

References 38

\section{Chapter 2 Transmission Line Components 41}

2.1 Fundamentals 41

2.2 Classification and Parameters 47

2.3 RF Coaxial Cables 51

2.4 Coaxial Connectors 55

2.5 Cable Assemblies 65

2.6 Waveguides and Flanges 66

2.7 Coaxial and Waveguide Components 68

2.8 Rotary Joints 73

Questions 75

References 75

\section{Chapter 3 Passive Components 77}

3.1 Substrates and Laminates 78

3.2 Resistors and Fixed Attenuators 84

3.3 Inductors, Chokes, and Transformers 90

3.4 Capacitors 96

3.5 EMI and RFI Filters 102

3.6 Power Dividers/Combiners, Splitters 107

3.7 Couplers 113

3.8 Beamformer Networks 118

3.9 Gain Equalizers 121

3.10 Circulators and Isolators 124

Questions 129

References 130 


\section{Chapter 4 Fixed Frequency Filters 131}

4.1 Fundamentals 131

4.2 Lumped LC-Element Filters 137

4.3 Cavity Filters 142

4.4 Ceramic Resonator Filters 143

4.5 Microstrip and Stripline Filters 146

4.6 Tubular Filters 147

4.7 Waveguide Filters 147

4.8 Yttrium-Iron Garnet (YIG) Filters 150

4.9 Thin- and Thick-Film Filters 154

4.10 Monolithic Crystal Filters 155

4.11 SAW and BAW Filters 157

4.12 MEMS Filters 160

4.13 Harmonic Filters 161

4.14 Frequency Multiplexers and Duplexers 164

4.15 Fixed Frequency Filter Assemblies 170

Questions 171

References 171

Chapter 5 Control Components: Attenuators, Phase Shifters, Time Delay Lines, and Controlled Frequency Filters 173

5.1 Fundamentals 173

5.2 Classification and Parameters 181

5.3 Variable Attenuators 184

5.3.1 Manually Controlled Attenuators 184

5.3.2 Electrically Variable Attenuators 185

5.3.3 Digitally Controlled Attenuators 190

5.3.4 Programmable Attenuators 193

5.3.5 Phase Invariant Attenuators 195

5.4 Phase Shifters 197

5.5 Time Delay Lines 201

5.6 Tunable and Switched Frequency Filters 206

Questions 209

References 209

Chapter 6 Control Components: Switches and Matrices 211

6.1 Fundamentals 211

6.2 Classification and Parameters 213

6.3 Solid-State Switches and Matrices 217

6.3.1 PIN-Diode Switches 218

6.3.2 FET/GaAs Switches 220

6.3.3 Solid-State Matrices 224 
6.4 Coaxial Electromechanical Switches and Matrices 227

6.5 Waveguide Electromechanical Switches 231

6.6 Microelectromechanical Switches 234

6.7 Ferrite Switches 239

6.8 Reed, Motorized, Redundancy, Shorting, Transmitter/Receiver, Bidirectional, and Programmable Switches 241

Questions 244

References 244

\section{Chapter 7 Amplifiers 247}

7.1 Fundamentals 247

7.2 Classification and Parameters 250

7.3 Low-Noise Amplifiers 255

7.4 High Dynamic Range Amplifiers 259

7.5 Solid-State Power Amplifiers 261

7.6 Wideband Solid-State Amplifiers 266

7.7 Variable Gain, Transimpedance, Limiting, Cryogenic, Distribution, Fast Recovering, and Temperature Compensated Amplifiers 268

7.8 Klystrodes, Klystrons, TWTs, Amplitrons, Crossed-Field, and Gyro-Amplifiers 272

7.9 Pulse Amplifiers 277

7.10 Intermodulation Distortion and Linearization of Power Amplifiers 277

Questions 282

References 282

\section{Chapter 8 Frequency Mixers and Converters 285}

8.1 Fundamentals 285

8.2 Classification and Parameters 289

8.3 Balanced Mixers 292

8.4 Image Reject Mixers 297

8.5 Harmonic Mixers 298

8.6 Frequency Converters 303

8.7 Self-Oscillating Mixers 305

Questions 308

References 309

\section{Chapter 9 Modulators and Demodulators 311}

9.1 Fundamentals 311

9.2 Parameters and Classification 316

9.3 Amplitude Modulators and Detectors 318

9.4 BPSK Modulators and Demodulators 322

9.5 I/Q Modulators and Demodulators 325

9.6 Vector Modulators 329 
9.7 Modulators with Extended Functional Possibilities 331

9.8 High-Speed Digital-to-Analog Converters in Modulators 332

9.9 Smoothing of Modulation Fronts 335

Questions 337

References 337

Chapter 10 Frequency Multipliers and Dividers 339

10.1 Fundamentals 339

10.2 Frequency Multipliers 344

10.2.1 Passive Frequency Multipliers 344

10.2.2 Active Frequency Multipliers 347

10.2.3 Harmonic Multipliers 349

10.2.4 Frequency Multipliers on PLL 350

10.3 Frequency Dividers 351

10.3.1 Prescalers 351

10.3.2 Counting Frequency Dividers 354

Questions 355

References 356

Chapter 11 Oscillation Sources 357

11.1 Fundamentals 357

11.2 Parameters 361

11.3 Standards of Frequency and Time 363

11.4 Crystal Oscillators 370

11.4.1 Clock Oscillators 373

11.4.2 High-precision Crystal Oscillators 376

11.4.3 Frequency-Controlled Crystal Modules 378

11.5 SAW and BAW Oscillators 379

11.6 MEMS Oscillators 382

11.7 Oscillators with Stabilized Resonators 382

11.8 Optoelectronic Oscillators 390

11.9 Millimeter-Wave on Gunn and IMPATT Diodes Oscillators 391

11.10 Oscillators with Sapphire-Loaded Cavities 398

11.11 Oscillators on Tubes Microwave 403

Questions 407

References 408

Chapter 12 Frequency Controlled Oscillators 411

12.1 Fundamentals 411

12.2 Classification and Parameters 413

12.3 Narrowband VCO MMICs and Modules 418

12.4 Wideband and Octave Tuned VCOs 423 
12.5 VCO with Crystal, SAW, Coaxial, and Dielectric Resonators 424

12.6 YIG-Tuned Oscillators 427

12.7 Digitally Tuned Oscillators 431

Questions 432

References 433

Chapter 13 Frequency Synthesizers, Signals, and Noise Sources 435

13.1 Fundamentals 435

13.2 Fixed Frequency Synthesizers 439

13.3 Direct Digital Synthesizers 443

13.4 Phase Locked Loop Frequency Synthesizers 449

13.5 Hybrid Synthesizers 454

13.6 Signal Synthesizers 460

13.7 Arbitrary Waveform and Noise Generators 466

Questions 471

References 471

Appendix A Web Sites and Names of Manufacturing Companies 473

\section{List of Acronyms 489}

\section{About the Authors 495}

\section{Index 497}

* Editor's Note: The brief summary and the contents of the books are reported as provided by the authors or the publishers. Authors and publishers are encouraged to send review copies of their recent books of potential interest to readers of Electronics to the Publisher (Dr. Shu-Kun Lin, Multidisciplinary Digital Publishing Institute (MDPI), Kandererstrasse 25, CH-4057 Basel, Switzerland. Tel. +41-61-683-77-34; Fax: +41-61-302-89-18; E-Mail: lin@mdpi.com). Some books will be offered to the scholarly community for the purpose of preparing full-length reviews.

\section{Note}

1. http://www.artechhouse.com/International/Books/Handbook-of-RF-Microwave-andMillimeterWave-Compon-1987.aspx.

(C) 2013 by the authors; licensee MDPI, Basel, Switzerland. This article is an open access article distributed under the terms and conditions of the Creative Commons Attribution license (http://creativecommons.org/licenses/by/3.0/). 